\title{
Lidil
}

Revue de linguistique et de didactique des langues

$59 \mid 2019$

Prononcer les langues : variations, émotions, médiations

\section{Enseignement de la grammaire dans les manuels de français langue étrangère et approche actionnelle}

Teaching Grammar in French As a Foreign Language Textbooks and Action-

Oriented Approach

\section{Margaret Bento}

\section{OpenEdition}

Journals

Édition électronique

URL : http://journals.openedition.org/lidil/6465

DOI : $10.4000 /$ lidil.6465

ISSN : 1960-6052

Éditeur

UGA Éditions/Université Grenoble Alpes

Édition imprimée

ISBN : 978-2-37747-090-7

ISSN : $1146-6480$

Référence électronique

Margaret Bento, «Enseignement de la grammaire dans les manuels de français langue étrangère et approche actionnelle », Lidil [En ligne], 59 | 2019, mis en ligne le 01 mai 2019, consulté le 10 décembre 2020. URL : http://journals.openedition.org/lidil/6465 ; DOI : https://doi.org/10.4000/lidil.6465

Ce document a été généré automatiquement le 10 décembre 2020.

(C) Lidil 


\section{Enseignement de la grammaire dans les manuels de français langue étrangère et approche actionnelle}

Teaching Grammar in French As a Foreign Language Textbooks and Action-

Oriented Approach

Margaret Bento

\section{Introduction}

1 Cette étude visera, à travers une analyse de manuels, à montrer quelle circulation méthodologique existe entre une approche actionnelle de la grammaire et sa mise en œuvre dans les manuels de français langue étrangère (dorénavant FLE).

2 Le Cadre commun de référence pour les langues (dorénavant CECRL) constitue le document de référence sur la conception de l'enseignement des langues en Europe. Les auteurs de manuels sont donc amenés à adapter leurs contenus à ces propositions. Bien que le CECRL ne préconise aucune méthodologie (Bourguignon, 2006; Frath, 2008; Bento, 2013), il donne tout de même des orientations didactiques de type actionnel. Les auteurs du Cadre précisent que

La compétence grammaticale est la capacité de comprendre et d'exprimer du sens en produisant et en reconnaissant des phrases bien formées selon ces principes et non de les mémoriser et de les reproduire comme des formules toutes faites. En ce sens, toute langue a une grammaire extrêmement complexe qui ne saurait, à ce jour, faire l'objet d'un traitement exhaustif et définitif. (Conseil de l'Europe, 2001, p. 89)

Ainsi, un apprentissage prônant la répétition serait à éviter. Il est souligné tout au long du CECRL que la compétence linguistique (phonétique, lexique, grammaire) se construit à travers la réalisation de tâches, autrement dit on ne fait pas de la grammaire pour de la grammaire, mais de la grammaire dans un but communicatif. Le CECRL propose différents types d'exercices formels pouvant être employés: textes lacunaires, construction de 
phrases sur un modèle donné, choix multiples, exercices de substitution dans une catégorie, combinaison de phrases, traduction de phrases de la L1 vers la L2, questions/ réponses entrainant l'utilisation de certaines structures, exercices de développement de l'aisance langagière centrés sur la grammaire, etc. (Conseil de l'Europe, 2001, p. 116).

Cette diversité d'exercices montre que la perspective retenue par le Cadre est résolument plurilingue. Les auteurs soulignent l'intérêt de la grammaire contrastive dans l'enseignement/apprentissage des langues (Conseil de l'Europe, 2001, p. 115).

En ce qui concerne les manuels, objet de mon propos, les auteurs du Cadre indiquent qu'il existe une grande variété entre eux quant à la prise en compte des éléments grammaticaux (Conseil de l'Europe, 2001, p. 108). D’ailleurs, une étude menée, il y a près de 10 ans, sur la place de la grammaire dans les manuels de langue étrangère, confirmait la grande diversité méthodologique proposée par les auteurs dans ces ouvrages (Bento, 2010). C'est cette variété ou non qui sera mise au jour dans mon analyse des manuels de FLE d'aujourd'hui.

5 La question se pose aussi de savoir comment faire de la grammaire dans une approche actionnelle. Dans quelle mesure les activités grammaticales présentées dans les manuels de français langue étrangère sont-elles en adéquation, ou non, avec une approche actionnelle ? Je me suis donc intéressée aux démarches grammaticales, non pas au niveau des contenus enseignés, mais au niveau de l'approche méthodologique employée.

\section{L'enseignement de la grammaire dans l'approche actionnelle}

6 Depuis l'avènement de l'approche communicative et encore plus aujourd'hui avec l'approche actionnelle, les ressources se sont diversifiées et le document dit "authentique", en tant que "tout message élaboré par les francophones pour les francophones à des fins de communication réelle [...]» (Cuq, 2003, p. 29), est en usage dans l'enseignement du FLE en Europe. Parallèlement à ce recours aux ressources authentiques, la notion de support a été remplacée par celle de documentation (Puren, 2013 et 2014). Cette logique «documentation» permet aux apprenants de réaliser des actions grâce à l'analyse ou à la constitution d'un dossier documentaire. Le terme de « documentation » est défini par Beauné, Bento et Riquois (2015) comme une « activité de recherche, de sélection et d'exploitation d'un ensemble de documents en vue de réaliser une action ». La comparaison de manuels édités ces vingt dernières années illustre l'évolution d'une logique de support vers une logique «documentation» (Puren, 2014; Bento, 2015). Ce n'est plus «l'unité de communication donnée par le dialogue de base " qui est visée mais «l'unité d'action » (Puren, 2010a, p. 4). C'est à partir «d'une série de documents (oraux, écrits ou iconiques)» que l'apprenant va développer «des compétences de compréhension » (Puren, 2010a, p. 6).

7 Ainsi, si on pousse le raisonnement jusqu'au bout, pour la grammaire, la logique "documentation" devrait conduire à collecter un ensemble de documents afin de trouver les règles grammaticales nécessaires pour accomplir des tâches. C'est ce qui permettra à l'apprenant de prendre une posture d'acteur social : il sélectionne, il cherche une information, il analyse, il croise des informations... comme cela se pratique dans sa vie quotidienne. Si la logique « documentation» est bien en place pour les activités de compréhension (Bento, 2015), qu'en est-il pour les activités grammaticales? 


\section{Corpus et méthodologie}

8 L'analyse porte sur 8 manuels de français langue étrangère publiés en France entre 2014 et 2017. Il s'agit des deux manuels les plus récents des quatre maisons d'édition qui publient des manuels de FLE en France :

- Saison et Édito chez Didier ;

- Nickel! et Tendances chez Clé International ;

- Entre nous et Défi chez Maison des langues ;

- Cosmopolite et Texto chez Hachette.

$9 \mathrm{Au}$ niveau méthodologique pour l'analyse des données recueillies, les démarches privilégiées par les auteurs pour l'enseignement de la grammaire ont été étudiées pour l'ensemble des manuels. La nature de l'enseignement de la grammaire se fonde sur différents types de démarche: explicite/implicite et déductive/inductive (entre autres, Pescheux, 2007, p.188-190; Bento, 2010). Une approche explicite de la grammaire suppose un enseignement où les règles grammaticales sont énoncées. Ces règles peuvent être données en amont par l'enseignant ou induites par l'élève grâce à des activités de conceptualisation, c'est-à-dire un travail de réflexion sur la langue. Un enseignement implicite de la grammaire suppose que l'enseignant ou l'apprenant n'émettra pas de règles, évitant ainsi l'utilisation du métalangage comme complément, démonstratif, etc. Une démarche déductive consiste à appliquer les règles de grammaire à travers des exercices. Enfin, une démarche inductive conduit l'apprenant à faire des hypothèses puis à dégager une règle (qu'elle soit explicite ou implicite) à partir de l'observation d'un ensemble de phrases comportant l'élément grammatical visé ou à partir de l'exécution d'exercices. Dans la pratique, certains de ces modes peuvent se combiner entre eux. Ainsi, une grammaire explicite et déductive consiste à énoncer une règle grammaticale avant de faire des exercices d'application, comme dans les approches traditionnelles. En général, les élèves sont actifs dans l'application des exercices, mais non dans la découverte de la règle qui est souvent donnée par l'enseignant. Une grammaire implicite et inductive suppose que les apprenants effectuent des exercices systématiques sans que la règle ne soit énoncée. Dans cette approche, l'apprenant doit compléter ou transformer des phrases à partir d'un exemple donné. Si cette démarche ne présente pas d'utilisation de métalangage, elle semble tout de même conduire l'apprenant à une forme de conceptualisation, c'est-à-dire à l'élaboration d'une règle grammaticale, mais celle-ci sera intériorisée. Dans une grammaire inductive et explicite, les apprenants sont invités à comprendre le fonctionnement d'une règle grammaticale à partir d'un ensemble de phrases comportant le point à travailler. Les élèves aboutissent à la formulation de la règle par le biais d'activités de réflexion sur la langue. Toujours au niveau méthodologique, j'analyserai aussi la place du français et des autres langues dans les manuels.

Il s'agira dans cette recherche de voir la place occupée par la grammaire dans les manuels en observant les démarches d'une part et en analysant les activités qui sont utilisées par les auteurs d'autre part. On s'interrogera également sur la place occupée par les autres langues que la langue cible dans les exercices de grammaire. Pour répondre à ces questionnements, la place des séquences grammaticales dans l'organisation générale du manuel, la formulation des règles grammaticales, le choix des exercices et leur 
formulation seront analysés. Les résultats de cette étude permettront de voir les choix effectués par les auteurs des manuels, en lien avec leur interprétation du CECRL.

\section{La place de la grammaire dans les manuels}

11 L'ensemble des méthodes analysées intègrent de la grammaire dans leurs leçons. Dans tous les manuels, sauf Saison, on trouve, pour chaque leçon, des encarts consacrés à un point grammatical avec, à la suite ou à la fin du dossier ou de l'ouvrage, des activités d'entrainement. Saison se démarque en proposant une observation de phrases, puis une page consacrée aux exercices grammaticaux et enfin une page consacrée aux règles de grammaire. Ces trois phases sont séparées à l'intérieur de l'unité. On remarque également dans la plupart des manuels (sauf Saison et Édito) un précis ou récapitulatif grammatical et des tableaux de conjugaison.

La grammaire est donc largement présente dans l'ensemble des méthodes observées. Les points grammaticaux évoqués sont toujours au service de l'objectif visé, autrement dit, la leçon de grammaire est utilisée comme un outil que l'apprenant doit maitriser non pas comme une fin en soi, mais pour accomplir une tâche finale. Par exemple, les prépositions et les adverbes de lieu sont travaillés dans le but de demander des renseignements sur un lieu, se situer dans l'espace, s'orienter (par exemple, Nickel!, p. 67-78 ; Tendances, p. 33-46). On voit que les auteurs se conforment aux préconisations du CECRL (Conseil de l'Europe, 2001, p. 89).

\section{Les démarches grammaticales privilégiées}

Les manuels ont été analysés en fonction des différents types de démarches grammaticales recensés ; le tableau ci-dessous récapitule les orientations majeures.

Tableau 1. - Type de démarche utilisé dans les manuels.

\begin{tabular}{|l|c|c|c|c|}
\hline & $\begin{array}{r}\text { Grammaire } \\
\text { explicite }\end{array}$ & $\begin{array}{r}\text { Grammaire } \\
\text { implicite }\end{array}$ & $\begin{array}{r}\text { Grammaire } \\
\text { déductive }\end{array}$ & $\begin{array}{c}\text { Grammaire } \\
\text { inductive }\end{array}$ \\
\hline Cosmopolite & + & & & + \\
\hline Texto & + & & + & + \\
\hline Nickel! & + & & + & + \\
\hline Tendances & + & + & + & + \\
\hline Entre nous & + & & + & + \\
\hline Défi & + & + & & + \\
\hline Saison & + & & & + \\
\hline Édito & + & & & + \\
\hline
\end{tabular}


La lecture de ce tableau montre très clairement qu'une grammaire explicite et inductive est adoptée par l'ensemble des auteurs de manuels, même si on constate, à la marge, que déduction et grammaire implicite les côtoient parfois. Les manuels présentent donc une certaine homogénéité au niveau de la démarche grammaticale adoptée. Le tableau cidessous montre plus précisément les démarches adoptées par les auteurs et leur diversité.

Tableau 2. - Type de démarche méthodologique utilisé dans chaque manuel.

\begin{tabular}{|l|c|l|l|l|}
\hline & $\begin{array}{c}\text { Observation } \\
\text { Conceptualisation } \\
\text { Réemploi } \\
\text { Règle }\end{array}$ & $\begin{array}{c}\text { Observation } \\
\text { Conceptualisation } \\
\text { Réemploi }\end{array}$ & $\begin{array}{c}\text { Observation } \\
\text { Règle } \\
\text { Réemploi }\end{array}$ & $\begin{array}{c}\text { Règle } \\
\text { Exemple } \\
\text { (Réemploi) }\end{array}$ \\
\hline Cosmopolite & + & & & \\
\hline Texto & + & & + & + \\
\hline Nickel! & + & & & + \\
\hline Tendances & + & & & \\
\hline Entre nous & + & & & \\
\hline Défi & + & & & \\
\hline Saison & + & & & \\
\hline Édito & + & & & \\
\hline
\end{tabular}

15 Une analyse plus fine des démarches grammaticales dans les manuels montre que les auteurs adoptent des méthodologies sensiblement différentes pour découvrir les règles de grammaire. Prenons trois manuels qui illustrent la diversité de ces démarches : Nickel!, Tendances et Saison.

La méthode Nickel! propose deux démarches différentes. La première consiste en une grammaire inductive et explicite avec:

- une observation de corpus ;

- une conceptualisation à partir de questions ;

- la règle grammaticale donnée immédiatement dans le texte ;

- des exercices d'application.

17 EXEMPLE 1 (Nickel!, p. 46)

Les articles partitifs

Observez ces phrases :

Je mange du pain, des tartines avec du beurre ou de la confiture et je bois du lait avec du cacao et parfois aussi des céréales.

À quoi ça sert ?

Les articles partitifs servent...

- à déterminer des noms « non comptables » :

Vous avez de l'huile d'olive? 
- à exprimer des quantités indéfinies :

Vous voulez des biscuits?

(une certaine quantité) d'un

Voilà du gâteau pour ton goûter.

(une partie de)

[...]

Complétez les phrases suivantes à l'aide d'un article partitif :

1. Achète ... fromage pour ce soir. [...]

\section{EXEMPLE 2 (Nickel !, p. 58)}

Les adjectifs possessifs ${ }^{1}$

Observez ces phrases :

On va avec Pauline retrouver nos copains.

Avec quel mot s'accorde l'adjectif possessif?

Maintenant observez le tableau ci-contre.

[tableau des adjectifs possessifs]

Dans quel cas les adjectifs possessifs ont-ils des formes différentes au masculin et au

féminin?

C'est pareil dans votre langue?

[...]

À quoi ça sert ?

Les adjectifs possessifs servent..

- à exprimer la possession :

Ton manteau est dans la voiture.

- à indiquer des habitudes :

Salut! Je file prendre mon bus.

Complétez avec des adjectifs possessifs :

1. Martine passe ... vacances avec ... famille. [...]

La deuxième démarche consiste également en une grammaire inductive et explicite avec :

- une observation de corpus ;

- des règles de grammaire (sans conceptualisation) ;

- des exercices d'application.

\section{EXEMPLE 3 (Nickel!, p. 82)}

Les adjectifs démonstratifs

Observez ces phrases :

Cette année, nous faisons une réduction de $5 \%$.

Ce gîte est à Saint-Jean-du-Gard même?

Est-ce que ces chambres sont au même étage?

Comme tous les déterminants, les adjectifs démonstratifs s'accordent en genre

et en nombre avec les noms qu'ils précèdent.

[tableau des adjectifs démonstratifs]

À quoi ça sert ?

Les adjectifs possessifs servent...

- à désigner des personnes ou des objets présents dans la situation :

Prends cette chaise, je m'assois sur le tabouret.

- à faire référence à des personnes ou à des objets déjà mentionnés :

Le 25 septembre? Oui pour cette date ça marche.

Complétez les phrases suivantes à l'aide d'un adjectif démonstratif :

2. J'aime beaucoup ... immeubles anciens. [...] 
Tendances propose une démarche différente de la précédente dans la mesure où la grammaire est inductive, mais plus ou moins implicite. En effet, pendant la leçon, la règle de grammaire n'est pas énoncée. On la trouve en fin d'unité :

- observation de corpus ;

- exercice d'application ;

- formalisation de la règle en fin d'unité.

Remarquons que le manuel Défi propose le même fonctionnement, mais que la règle y est donnée dans le précis grammatical.

EXEMPLE 4 (Tendances, p. 49)

Réfléchissons... Les adjectifs possessifs

Observez les mots en gras. Qu'est-ce qu'ils expriment ?

Greg: Tiens, tes ciseaux.

Mélanie: Sur la photo, c'est mon père.

Les mots en gras sont des adjectifs possessifs.

Complétez le tableau des adjectifs possessifs avec l'aide du professeur :

\begin{tabular}{|l|c|c|c|c|}
\hline Personne qui possède & Je & Tu & Vous & Il/Elle \\
\hline Masculin singulier & mon père & & votre père & \\
\hline Féminin singulier & & & votre mère & \\
\hline Pluriel & & tes ciseaux & vos enfants & \\
\hline
\end{tabular}

Complétez avec un adjectif possessif :

Ludovic montre des photos à Mélanie.

Ludovic : Regarde. Là, c'est ... rue à Bruxelles. Ici, c'est ... appartement. Et ici, ... université. [...]

On peut remarquer que dans cette activité de réflexion sur la langue, l'apprenant n'est pas autonome puisque le corpus présenté n'est pas suffisant pour lui permettre de remplir le tableau. Il aura sans doute besoin de l'enseignant, comme indiqué dans la consigne, pour accomplir cette tâche. En revanche, l'exemple permet à l'apprenant d'être totalement autonome dans la conceptualisation.

Le troisième manuel, Saison, propose une grammaire inductive et explicite avec une observation liée à un travail de conceptualisation, mais la règle est énoncée dans le précis grammatical qui se trouve à la fin de l'ouvrage. La démarche comporte :

- une observation de corpus ;

- une conceptualisation guidée ;

- des exercices de systématisation presque en fin de manuel ;

- la formalisation de la règle en fin d'unité.

\section{EXEMPLE 5 (Saison, p. 60 et 65)}

Observez ces phrases (p. 60):

Nous faisons de la randonnée. Je fais du VTT le samedi. Je joue au tennis.

a. Dans chaque phrase, dites si le sport est au féminin ou au masculin.

b. Soulignez le mot qui est placé après les verbes.

c. Remplacez VTT et tennis par pétanque. Que remarquez-vous?

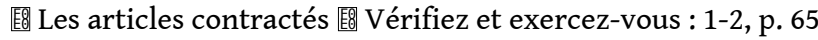

Les articles contractés (p. 65) : 


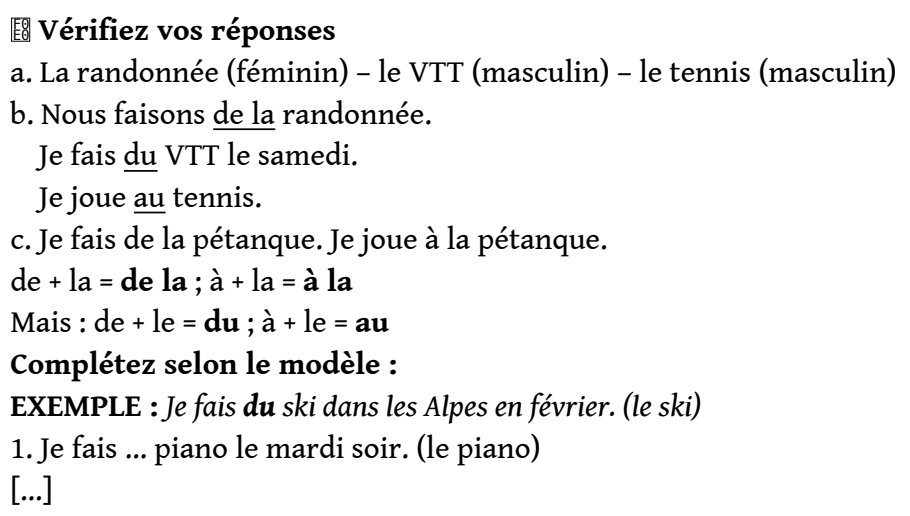

Au final, ces différents exemples attestent d'une diversité des démarches toute relative. En effet, tous les auteurs proposent une grammaire inductive et explicite, même si on trouve quelques exemples de grammaire implicite et déductive disséminés dans certains manuels. En revanche, on remarque que la conscientisation du fonctionnement de la langue se fait de différentes manières. Même si les auteurs amènent les apprenants à comprendre par eux-mêmes les règles grammaticales en proposant des activités de conceptualisation par le biais de l'observation d'une série d'exemples et de questions choisies, les formulations des règles sont fournies plus ou moins tôt dans la leçon. Certains auteurs donnent la règle tout de suite après la question permettant le travail de conceptualisation, d'autres la donnent en fin de leçon et d'autres encore dans le précis grammatical à la fin du manuel. On peut imaginer que lorsque la règle est donnée à côté de la question de repérage, les apprenants peuvent se référer à la règle plus facilement, avant même de faire le travail de conceptualisation rendant ainsi la démarche déductive et explicite pour les apprenants.

\section{Les exercices grammaticaux}

Les exercices qui accompagnent ces démarches sont assez peu diversifiés. On trouve essentiellement des textes lacunaires (cf. exemples supra). On relève plus rarement des exercices de construction de phrases sur un modèle donné, des exercices de substitution et des questions/réponses entrainant l'utilisation de certaines structures. On retrouve ici le listage des types d'exercices classiques indiqué dans le CECRL (Conseil de l'Europe, 2001, p. 116). Il manque cependant les exercices de traduction de phrases de la L1 vers la L2, mais c'est sans aucun doute dû au type même de manuels auquel nous avons affaire ici, qui est destiné à des publics très hétérogènes. Quoi qu'il en soit, les exercices proposés sont «classiques» et ne vont pas dans le sens d'une logique «documentation». Le processus n'incite pas les apprenants à collecter eux-mêmes les documents ou l'enseignant à rassembler un ensemble de documents permettant à l'apprenant de chercher l'information dont il a besoin pour accomplir les tâches comme un acteur social.

\section{La place des langues dans les activités grammaticales}

Les auteurs des manuels proposent relativement peu de réflexions sur la langue source des apprenants pour travailler la conceptualisation grammaticale. On en trouve quelques rares exemples dans Entre nous et Défi, mais le manuel Nickel! est le seul à proposer très 
régulièrement, dans les points dédiés à la grammaire, des questions sur le fonctionnement de la langue de l'apprenant. Mais l'ensemble de ces propositions ne vont pas plus loin qu'une simple question sur l'existence du point langue envisagé. manuels et peut être utilisée comme une aide à la compréhension de la grammaire, mais cela reste marginal. Le fait que les manuels soient édités en France et donc destinés à des publics hétérogènes linguistiquement n'incite sans doute pas les auteurs à s'appuyer sur cette possibilité. Pourtant, les comparaisons interlinguales (Roulet, 1980; Dabène \& Degache, 1996; Castellotti, 2001; Moore, 2006), permettent aux apprenants la mise en œuvre d'opérations de transfert de connaissances où ils s'appuient sur leurs langues connues pour comprendre la langue ciblée par l'apprentissage. Il s'agit pour eux d'accéder à la compréhension du fonctionnement du français tout en réfléchissant sur le fonctionnement des langues qu'ils connaissent. Cette compréhension passe par l'observation des similitudes et des différences entre les langues. Cette possibilité est largement ignorée par les auteurs des manuels.

\section{Conclusion}

Cette étude a permis de répondre à plusieurs questions. La première concernait la place de la grammaire dans les manuels FLE étudiés. On a pu constater que les auteurs lui donnent une place réelle et importante. Il s'est agi ensuite de se questionner sur les démarches et activités privilégiées par les auteurs. Au niveau méthodologique, forme et sens ne sont jamais dissociés lors de l'explication grammaticale. Il apparait aussi que les rédacteurs de manuels proposent prioritairement une grammaire inductive et explicite avec un schéma du type : repérage, reconnaissance, conceptualisation des faits de langue, application et entrainement. Cette mise en œuvre suit les principes du constructivisme et $\mathrm{du}$ socioconstructivisme décrits par les didacticiens pour les approches actionnelles (Bento, 2013) et favorisant l'activité de l'apprenant (Griggs, 2009, p. 84 ; Narcy-Combes \& Walski, 2004, p. 35). Cette démarche s'accompagne aussi d'approches déductives et implicites, mais de manière marginale. L'ensemble des exercices grammaticaux sont relativement peu diversifiés, on relève essentiellement des exercices lacunaires. Ces exercices permettent la réalisation de tâches communicatives en lien avec les thématiques des leçons, rendant ainsi le travail grammatical fortement contextualisé et donc porteur de sens par rapport aux tâches finales. Enfin, sur la question de l'utilisation d'autres langues que la langue cible dans les manuels, la plupart des auteurs semblent hésiter à recourir à la langue source des apprenants pour faire comprendre un fonctionnement grammatical ou encore le sens d'une structure. Ainsi, ils adoptent peu le 
point de vue duCECRL sur les avantages de la comparaison interlinguale et de la grammaire contrastive.

Les résultats de cette analyse montrent une certaine uniformisation de l'approche grammaticale pratiquée: grammaire inductive et explicite, peu contrastive, exercices lacunaires, contextualisés. L'éclectisme signalé par le CECRL ne semble plus de mise comme nous le verrons plus tard:

[...] de toute évidence, il existe des variations de réaction considérables selon l'âge, la nature et l'origine des apprenants quant aux éléments auxquels ils répondent de la manière la plus productive; ces mêmes variations se retrouvent parmi les enseignants, les auteurs de méthodes, etc., quant à l'équilibre des éléments qu'ils introduisent dans un cours, selon l'importance qu'ils attachent à la production plutôt qu'à la réception, à la correction plutôt qu'à l'aisance, etc. (Conseil de l'Europe, 2001, p. 108)

Il est difficile de connaitre les raisons de cette uniformisation, mais peut-être est-ce tout simplement, pour les auteurs, l'application d'une approche grammaticale qui fonctionne auprès des apprenants. Nous sommes là face à une évolution méthodologique évidente. En effet, une analyse de manuels publiés entre 2006 et 2008 montrait un enseignement plus éclectique de la grammaire où les phrases d'apprentissage inductives, déductives, implicites et explicites alternaient largement. Je constatais que l'actualisation de la démarche grammaticale était «parfois d'inspiration socioconstructiviste, parfois plus traditionnel ou éclectique » (Bento, 2010, p. 83).

Le schéma grammatical que nous relevons régulièrement dans les manuels d'aujourd'hui a été initié par Besse en 1964, puis par Besse et Porquier en 1984 et a été qualifié de standard par Puren en 2010 (p. 30-31). Il semble, en effet, bien installé aujourd'hui. On constate une nette évolution vers cette standardisation. On voit donc que la logique « documentation » en place actuellement pour les activités de compréhension ne sont pas du tout d'actualité pour les activités grammaticales dans les manuels. Ainsi, il y a une faille dans l'unité d'action, actuellement essentiellement dirigée vers des compétences de compréhension, qui satellise les compétences grammaticales et plus largement linguistiques. L'enseignement grammatical au final se standardise sous une forme dont on ne peut nier l'inspiration socioconstructiviste, puisque l'apprenant est en action et formule des règles grâce à une réflexion sur la langue, mais également applicationniste puisque l'apprenant exécute des activités dont il n'a pas l'initiative.

\section{BIBLIOGRAPHIE}

BEAUnÉ, Aurélie, Bento, Margaret \& Riquois, Estelle. (2015). The Authenticity of Resources for the Teaching of Foreign Languages and Cultures in France: Variable Geometry Notion. IARTEM eJournal, 7(3), 1-24. Disponible en ligne sur <http://biriwa.com/iartem/ejournal/>.

BENTO, Margaret. (2010). L'enseignement de la grammaire dans les manuels de langues vivantes au collège en France face aux instructions officielles et au CECRL. Les langues modernes, 3, p. 71-83. 
BENTO, Margaret. (2013). Regards théoriques sur la perspective actionnelle dans l'enseignement des langues en France. Éducation \& Didactique, 6(3), p. 95-107.

BENTO, Margaret (2015). Les ressources en classe de FLE/FLS : du support à la documentation authentique. Dans J.-M. Defays et coll. (dir.), 20 ans de FLES - Faits et gestes de la didactique du français langue étrangère et seconde de 1995 à 2015 (vol. 3, 13-18) Bruxelles : EME.

BESSE, Henri. (1964). Les exercices de conceptualisation ou la réflexion grammaticale au niveau 2. Voix et Images du CRÉDIF, 2, 38-44.

BESSE, Henri \& PORQUIER, Rémi. (1984). Grammaires et didactique des langues. Paris : CREDIF-Hatier. BOURGUIGNON, Claire. (2006). La responsabilité des enseignants de langues à l'aune du Cadre européen commun de référence. Langues modernes. Disponible en ligne sur $<w w w . a p l v-$ languesmodernes.org/article.php3?id_article=655>.

CASTELLOTTI, Véronique. (2001). La langue maternelle en classe de langue étrangère. Paris : Clé International.

CONSEIL DE L'EUROPE (2001). Cadre commun européen de référence pour les langues. Paris : Didier.

CUQ, Jean-Pierre. (2003). Dictionnaire de didactique du français langue étrangère et seconde. Paris : Clé International.

DABÈnE, Louise \& DEGACHE, Christian. (1996). Comprendre les langues voisines. Études de linguistique appliquée, 104.

FRATH, Pierre. (2008). Le Cadre européen commun de référence pour les langues et le Portfolio européen des langues: où en sommes-nous? Langues modernes, 2, 11-18.

GRIGGS, Peter. (2009). À propos de l'articulation entre l'agir de l'usage et l'agir de l'apprentissage dans une approche actionnelle : une perspective sociocognitive. Dans L'approche actionnelle dans l'enseignement des langues (p. 80-100). Barcelone : Maison des langues.

MOORE, Danièle. (2006). Plurilinguismes et école. Paris : Didier.

NARCY-COMBES, Jean-Paul \& WALSKI, Jennifer. (2004). Le concept de tâches soumis au crible de nouvelles questions. Les cahiers de l'APLIUT, XXIII(1), 27-44.

PESCHEUX, Marion. (2007). Analyse de pratique enseignante en FLE/S : Memento pour une ergonomie didactique en FLE. Paris : L'Harmattan.

PUREN, Christian. (2010a). La didactique des langues-cultures comme domaine de recherche. Disponible en ligne sur <www.christianpuren.com/cours-la-dlc-comme-domaine-de-recherche/>.

PUREN, Christian. (2010b). Avant-propos. Dans M. Denyer et coll., Version originale 2. Paris : Éditions Maison des langues.

PUREN, Christian. (2013). Les documents, supports pour enseigner. Les cahiers pédagogiques, 508, $19-20$.

PUREN, Christian. (2014). L'exploitation didactique des documents authentiques en didactique des langues-cultures : trois grandes « logiques » différentes. Disponible sur <www.christianpuren.com/ mes-travaux/2014c/>.

ROULET, Eddy. (1980). Langue maternelle et langues secondes vers une pédagogie intégrée. Paris : HatierCrédif.

Bibliographie des manuels 
Auge Npai, Hélène, Marlhens, Claire, CAnada Pujols, Maria-Dolores \& Martin, Llucia. (2014).

Nickel! 1. Paris : Clé International.

Braud, Céline, Calvez, Aurélien, Cornuau, Guillaume, Jacob, Aanne, Vidal, Sandrine, Pinson, Cécile \& AlCARAz, Marion. (2016). Édito 1. Paris : Didier.

Chahi, Fatiha, Denyer, Monique, Gloanec, Audrey, Briet, Geneviève, Neuenschwander, Valérie \& F ouILLET, Raphaëlle. (2018). Défi 1. Paris : Maison des langues.

GirARDET, Jacky, PÉCheur, Jacques, GibBE, Colette, PARIZET, Marie-Louise. (2016). Tendances. Paris : Clé International.

HIRSCHSPRUNG, Nathalie \& TRICOT, Tony. (2017). Cosmopolite 1. Paris : Hachette.

LE BOUGNEC, Jean-Thierry \& LoPES, Marie-Josée. (2016). Texto 1. Paris : Hachette.

Pruvost, Neige, Courteaud, Frédérique, Gomez-Jordana, Sonia, Blondel, François, Chahi, Fatiha, C aballero, Ginebra, Poisson-Quinton, Sylvie, Daupras, Cindy, Delannoy, Gaëlle \& Brandel, Katia.

(2015). Entre nous 1. Paris : Maison des langues.

RiPAud, Delphine, CRos, Isabelle, Heu-Boulaht, Émilie, DupleIX, Dorothée \& KaSAZIAN, Émilie.

(2015). Saison 1. Paris : Didier.

\section{NOTES}

1. Bien que ce ne soit pas le propos de cet article, nous pouvons nous interroger sur l'obsolescence de la terminologie métalinguistique utilisée dans les manuels. Alors qu'aujourd'hui nous parlons plutôt de déterminants possessifs ou démonstratifs, nous trouvons encore ici les termes de « adjectifs possessifs » ou plus loin de « adjectifs démonstratifs ».

\section{RÉSUMÉS}

Les auteurs de manuels reprennent les préconisations du Cadre européen commun de référence pour les langues (CECRL) pour déterminer leurs contenus et se placent aujourd'hui dans une approche actionnelle. Cet article montre, à partir de l'analyse de 8 manuels de français langue étrangère pour adultes et grands adolescents, que les auteurs suivent un schéma qui devient classique pour la démarche d'enseignement/ apprentissage de la grammaire. En effet, les auteurs proposent une grammaire inductive et explicite dans le cadre d'activités souvent contextualisées au service de tâches communicatives, mais qui ne relèvent pas d'une logique "documentation » comme on pourrait s'y attendre dans une approche actionnelle.

Textbook authors use the recommendations of the Common European Framework of Reference for Languages (CEFR) to determine their contents and they adopt an action-oriented approach. This article shows, from the analysis of 8 French as a foreign language textbooks for adults and adolescents, that the authors follow a pattern which becomes classical for the grammar teaching approach. Indeed, the authors propose an inductive and explicit grammar in the framework of activities often contextualized in the service of communicative tasks. However, these activities 
do not correspond to a "documentation" logic as one would expect in an action-oriented approach.

INDEX

Keywords : modern language course books, the teaching of grammar, official instructions, CEFR, authors of course books

Mots-clés : manuels de français langue étrangère, enseignement de la grammaire, CECRL, auteurs de manuels, approche actionnelle

\section{AUTEUR}

MARGARET BENTO

Laboratoire Éducation, discours, apprentissages (EDA), Université Paris Descartes 